\title{
Two Radio Pulsars with Magnetar Fields
}

\author{
M. A. McLaughlin, D. R. Lorimer, A. G. Lyne, \\ M. Kramer, A. J. Faulkner \\ Jodrell Bank Observatory, Macclesfield, UK \\ V. M. Kaspi \\ McGill University, Montreal, Canada
}

I. H. Stairs

University of British Columbia, Vancouver, Canada

R. N. Manchester, G. Hobbs

ATNF - CSIRO, Epping, Australia

F. Camilo

Columbia University, New York NY, USA
A. Possenti \& N. D'Amico
Osservatorio Astronomico di Cagliari, Capoterra, Italy

\begin{abstract}
PSRs J1847-0130 and J1718-37184 have inferred surface dipole magnetic fields greater than those of any other known pulsars and well above the "quantum critical field" above which some models predict radio emission should not occur. These fields are similar to those of the anomalous X-ray pulsars (AXPs), which growing evidence suggests are "magnetars". The lack of AXP-like X-ray emission from these radio pulsars (and the non-detection of radio emission from the AXPs) creates new challenges for understanding pulsar emission physics and the relationship between these classes of apparently young neutron stars.
\end{abstract}

\section{Results}

Both of these pulsars were discovered in the Parkes Multibeam Pulsar Survey (see e.g., Manchester et al. 2001). PSR J1847-0130 has a spin period of $6.7 \mathrm{~s}$ and inferred surface dipole magnetic field ${ }^{1}$ of $9.4 \times 10^{13}$ G. PSR J1718-37184 has a period of $3.4 \mathrm{~s}$ and magnetic field of $7.4 \times 10^{13} \mathrm{G}$. The magnetic fields of both

\footnotetext{
${ }^{1}$ Calculated via the standard magnetic dipole formula (Manchester \& Taylor 1977), $B=3.2 \times$ $10^{19} \sqrt{P \dot{P}} \mathrm{G}$, where $P$ is in seconds.
} 
pulsars are well above the "quantum critical field" $\simeq 4.4 \times 10^{13} \mathrm{G}$ above which some models predicted radio emission should not occur (Baring \& Harding 1998). Both pulsars have average radio luminosities, indicating that photon splitting does not suppress pair production at these magnetic field strengths.

In Table 1, we compare the spin-down properties, distances and X-ray luminosities of the high-field pulsars and the AXPs. Using archival ASCA data, we measure an upper limit to the X-ray luminosity of PSR J1847-0130 that is lower than the luminosities of all but one AXP. We have analyzed a Chandra observation with PSR J1718-37184 in the field and detect the pulsar with a soft, thermal spectrum and with an X-ray luminosity much lower than that of any of the AXPs. Hydrogen atmosphere spectral fits yield a temperature that is consistent with standard neutron star cooling curves (Kaminker, Haensel \& Yakovlev 2001).

Table 1. PSR/AXP spin parameters, distances and luminosities.

\begin{tabular}{lcccc}
\hline Name & $\begin{array}{c}P \\
(\mathrm{~s})\end{array}$ & $\begin{array}{c}B \\
\left(10^{14} \mathrm{G}\right)\end{array}$ & $\begin{array}{c}D \\
(\mathrm{kpc})\end{array}$ & $\begin{array}{c}L(2-10 \mathrm{keV}) \\
\left(10^{33} \mathrm{ergs} \mathrm{s}^{-1}\right)\end{array}$ \\
\hline 1E 1048.1-5937 & 6.5 & 5.0 & $\geq 2.7$ & $\geq 5$ \\
1E 2259+586 & 7.0 & 0.59 & $4-7$ & $40-100$ \\
4U 0142+61 & 8.7 & 1.3 & $\geq 1.0$ or $\geq 2.7$ & $\geq 10$ or $\geq 70$ \\
RXS J170849-400910 & 11.0 & 4.6 & $\sim 8$ & $\sim 500$ \\
1E 1841-045 & 11.8 & 7.1 & $5.7-8.5$ & $20-50$ \\
\hline PSR J1718-37184 & 3.4 & 0.74 & $3-5$ & 0.0009 \\
PSR J1847-0130 & 6.7 & 0.94 & $6-11$ & $<3-8$ \\
\hline
\end{tabular}

It is unclear how the high-field pulsars and AXPs can have such similar spin-down parameters but such different emission properties. One possibility is that high-field pulsars and AXPs have similar dipole magnetic fields but AXPs have quadrupole (or higher) components. Finding more high-field radio pulsars is essential for understanding the relationship between these two populations and constraining the pulsar emission mechanism. Because of selection effects against the detection of long-period radio pulsars, there may be many more of these objects than are currently known. Finally, the discovery of radio emission from these two pulsars shows that there is no reason, a priori, why the AXPs cannot be radio emitters. While searches for radio emission from the currently known AXPs have so far been unsuccessful or unconfirmed (but see Malofeev et al., these proceedings), this may simply be the result of unfavorable beaming.

\section{References}

Baring, M. G., \& Harding, A. K., 1998, ApJ, 507, L55

Kaminker, A. D., Haensel, P., \& Yakovlev, D. G. 2001, A\&A, 373, L17

Manchester R. N., \& Taylor J. H. 1977, Pulsars, (San Francisco: Freeman)

Manchester R. N. et al. 2001, MNRAS, 328, 17

McLaughlin, M. A. et al. 2003, ApJ, 591, L135 